\title{
Aprendizaje-Servicio: una práctica educativa innovadora que promueve el desarrollo de competencias del estudiantado universitario
}

Service-Learning: an innovative educational practice promoting competence development in university students

\section{Volumen 18, Número 3 \\ Setiembre-Diciembre}

pp. 1-22

\section{Este número se publica el 1 de setiembre de 2018 \\ DOI: https://doi.org/10.15517/aie.v18i3.34418}

\author{
Domingo Mayor Paredes
}

Revista indizada en REDALYC, $\underline{\text { SCIELO }}$

Revista distribuida en las bases de datos:

LATINDEX, DOAJ, $\underline{\text { REDIB }}$ IRESIE, CLASE, DIALNET, SHERPA/ROMEO, QUALIS-CAPES, MIAR

Revista registrada en los directorios:

ULRICH'S, REDIE, RINACE, OEI, MAESTROTECA, PREAL, $\underline{\text { CLACSO }}$ 


\title{
Aprendizaje-Servicio: una práctica educativa innovadora que promueve el desarrollo de competencias del estudiantado universitario
}

\author{
Educational Approach to Sexuality in students with intellectual disability in a Costa Rican \\ secondary education institution
}

\section{Domingo Mayor Paredes ${ }^{1}$}

\begin{abstract}
Resumen: Este artículo presenta algunos de los resultados de una investigación centrada en el análisis de la influencia que tienen los proyectos de Aprendizaje-Servicio (ApS) en el desarrollo de diversas competencias del estudiantado universitario. El estudio tomó como eje de indagación un proyecto de ApS que estaba implementando el alumnado universitario, en el marco de una de las asignaturas del grado de Educación Social, en la residencia de un Instituto de Enseñanza Secundaria. En el mismo participaron: estudiantado y profesor universitario, alumnado de secundaria y dos educadoras sociales del Instituto. Para ello se utilizó el estudio de caso como metodología de la investigación y el análisis de documentos, la observación participante, las entrevistas semiestructuradas grupales y un cuestionario ad hoc, diseñado por el profesor universitario, como estrategias para la recogida de información. Los resultados obtenidos permiten constatar los efectos producidos en distintas dimensiones relacionadas con las competencias personales-estudiantiles y sociales: interés, autonomía, autovaloración, conexión teoría-prácticas, reflexión y en la satisfacción del estudiantado que participó en el mismo. Estos hallazgos enfatizan la importancia de esta metodología para la adquisición de competencias personales-estudiantiles y sociales del alumnado universitario.
\end{abstract}

Palabras clave: Aprendizaje-Servicio, desarrollo de competencias, innovación pedagógica, estudiantes universitarios.

\begin{abstract}
This article presents some of the results of a study focused on the analysis of the influence of ServiceLearning (SL) projects on the development of university student competencies. The axis of the study was inquiry into an SL project implemented by university students in a high school residence in the framework of one of their Social Education degree courses. University students and professors, high school students and two of the high school's social teachers participated in the project. The research methodology was the case study, with document analysis, participant observation, semi-structured group interviews and an ad hoc questionnaire designed by the university professor as information collecting strategies. The results found the effects of the various dimensions related to the personal-student and social competencies to be: interest, self-sufficiency, self-assessment, connection of theory and practice, reflection and satisfaction of the students who participated in it. These findings emphasize the importance of this methodology for university students in acquiring personal-student and social competencies.
\end{abstract}

Key words: Service-Learning, competence development, teaching innovation, university students.

1 Universidad de Extremadura, España. Doctorado en
Educación.

Dirección electrónica: domingomp@uex.ex

Artículo recibido: 29 de marzo, 2018

Enviado a corrección: 19 de julio, 2018

Aprobado: 27 de agosto, 2018 


\section{Introducción ${ }^{2}$}

En el nuevo escenario social, globalizado e influenciado significativamente por las tecnologías de la información y la comunicación se están produciendo cambios acelerados en los distintos ámbitos de la vida que están afectando a la forma de pensar, sentir y actuar de los individuos y grupos humanos, así como a la institución universitaria. En el contexto europeo, este proceso se inicia en 1999 con la creación del Espacio Europeo de Educación Superior $^{3}$ (EEES) con la intencionalidad de promover la Europa del conocimiento como factor clave para el desarrollo social y humano de la ciudadanía.

La integración de la educación superior en el EEES obligó a replantear diversos asuntos y a iniciar algunas modificaciones y adaptaciones en el sistema universitario europeo. Algunas de los asuntos afectan a la organización y contenidos de enseñanza, otros a la metodología a utilizar a la hora de planificar los procesos de enseñanza. La tarea es adecuar los métodos de enseñanza y aprendizaje al objetivo de adquisición de competencias para la vida (Comisión Europea, 2010; Ministerio de Educación, Cultura y Deporte, 2006).

Estos acuerdos se han concretado en la incorporación de un enfoque educativo centrado en el aprendizaje de competencias. La adopción de este enfoque comporta un cambio importante desde el punto de vista pedagógico y didáctico que afecta a la formación universitaria y a la práctica docente. Con él se pretende poner en marcha programas educativos sustentados en perfiles profesionales, revalorizar la formación práctica, impulsar nuevos roles docentes centrados en la orientación y seguimiento del aprendizaje, introducir metodologías activas que favorezcan el papel activo del alumnado en su formación, incorporar nuevos sistemas de evaluación, etc. Mudanzas que comportan un desafío para la cultura institucional y profesional arraigada en la universidad española.

En opinión de Pérez (2012), su adopción representa un enriquecimiento en la medida en que supone integrar y movilizar distintos tipos de aprendizajes (conocimientos, habilidades, actitudes y valores) para afrontar situaciones y problemas en contextos determinados. Favorece así, aprender autónomamente, autorregular y dirigir el propio

\footnotetext{
${ }^{2}$ Este artículo forma parte de un proyecto de investigación denominado: Ecologías del aprendizaje en contextos múltiples: análisis de proyectos de educación expandida y conformación de ciudadanía (ECOEC), financiado por el Ministerio de Economía y Competitividad. Proyecto I+D de Excelencia. Secretaria de Estado de Investigación, Desarrollo e Innovación. Dirección General de Investigación Científica y Técnica. Referencia: EDU2014-51961-P, dirigido por Juan Bautista Rodríguez Martínez, de la Facultad de Educación de la Universidad de Granada, como investigador principal.

${ }^{3}$ El EEES es el referente para la articulación de la formación universitaria de validez para el conjunto de la Unión Europea, con especificidad común de arquitectura de títulos, estructura, titulaciones, perfiles profesionales, certificación, homologación, movilidad, etc.
} 
aprendizaje y continuar aprendiendo a lo largo de la vida. Este potencial formativo conferido a las competencias viene acompañado de un mayor protagonismo del estudiante en su proceso de formación y de un cambio significativo en las propuestas educativas y en la propia cultura docente universitaria. Para De Miguel (2006), el enfoque por competencias invita al establecimiento de compromisos conjuntos en todo lo relativo al diseño, desarrollo y evaluación curricular, incorporando nuevas claves en la docencia universitaria.

Sin embargo, su implantación en la educación superior no está exenta de polémicas (Coll, 2007), motivadas por el diseño de nuevos planes de estudios sustentados en perfiles profesionales, la relevancia conferida a los logros, los nuevos roles docentes y su escasa formación, las dificultades para generar contextos reales que favorezcan la adquisición y desarrollo de competencias, así como la creación de pruebas para evaluar el nivel de competencias alcanzadas.

Para la puesta en acción del enfoque centrado en el aprendizaje por competencias hay que tomar decisiones relacionadas, por un lado, con la organización y contenidos de enseñanza y, por otro, con la metodología a utilizar a la hora de planificar los procesos de enseñanza. La tarea es adecuar los métodos de enseñanza y aprendizaje al objetivo de adquisición de competencias para la vida (Comisión Europea, 2010). En dicho proceso el profesorado diseña procesos de aprendizaje que permitan al estudiantado desarrollar estrategias analíticas y críticas, reflexivas y creativas, aprender a resolver problemas, etc.

Frente a las posiciones clásicas centradas en el aula y la actividad del profesorado, en la actualidad se considera prioritario organizar la enseñanza utilizando métodos que promuevan la actividad del alumno -individual y en grupo- y que permitan que el estudiantado alcance las competencias que se proponen como metas de aprendizaje (Imbernón y Medina, 2008; Rubio y Escofet, 2017). En este sentido se vienen implementando diferentes métodos: aprendizaje orientado por proyectos, aprendizaje basado en problemas, estudio de caso, aprendizaje colaborativo, Aprendizaje-Servicio, etc., que ponen el énfasis en la resolución de problemas, en el descubrimiento de nuevos aprendizajes, en el establecimiento de nuevas relaciones e interconexiones entre los contenidos, etc., favoreciendo el proceso de construcción de conocimientos relevantes, significativos y motivadores para el estudiantado.

De los métodos señalados anteriormente nos vamos a detener, en el epígrafe relacionado con el marco teórico, en describir y analizar la metodología del ApS o aprender haciendo, ya que ha sido la metodología utilizada en la experiencia investigada. 
En este escenario complejo y cambiante, la investigación estuvo focalizada en el análisis de un proyecto de ApS implementado por el estudiando universitario que perseguía los siguientes objetivos: a) Indagar las características de la organización donde se va a implementar el proyecto; b) Desarrollar actividades que promuevan la convivencia positiva entre el alumnado de secundaria; y c) Difundir los resultados de la investigación realizada y de la experiencia vivida entre el alumnado de la Facultad y distintos agentes sociales. Como preguntas de investigación se plantearon: a) Indagar la influencia del proyecto de ApS en el desarrollo de competencias del alumnado universitario; y b) Conocer las razones que sustentan la participación del estudiantado.

Desde una perspectiva de tipo etnográfico, este estudio se realizó en las aulas de la Facultad de Formación del Profesorado-Cáceres-España y en distintos espacios de la Residencia del Instituto de Enseñanza Secundaria “Universidad Laboral”. En este trabajo se presentan los resultados relacionados con el primer objetivo mencionado anteriormente.

\section{Marco teórico}

\subsection{ApS: una metodología innovadora que promueve el desarrollo de competencias en contextos reales}

El ApS, como metodología pedagógica, se sustenta en el aprendizaje experiencial (Dewey, 1950) y el servicio a la comunidad (James, 1910). Se diferencia de otras prácticas educativas experienciales (voluntariado, trabajo de campo, acciones comunitarias esporádicas, etc.) por vincular los objetivos de aprendizaje y los del servicio a la comunidad en un solo proyecto intencionalmente planificado en el que los participantes, concebidos como protagonistas de su proceso formativo, ponen en acción los saberes que conforman las competencias (conocimientos, habilidades, actitudes y valores) con el propósito de dar respuestas a necesidades sentidas por las comunidad. Ello requiere pensar en el alumnado no como proyecto de futuro, sino como un ser que se constituye siendo en relación con otros seres humanos en distintos contextos educativos.

El significante ApS, como objeto de estudio de naturaleza multidimensional y multidisciplinar, está sirviendo como categoría de análisis para una plétora de estudios y ensayos teóricos focalizados en indagar múltiples dimensiones vinculadas a su evolución histórica en distintos contextos socioculturales, marcos teóricos que lo sustentan, dinamismos pedagógicos que lo configuran, impacto en los agentes socioeducativos que participan en su materialización, proceso metodológico que articula las prácticas, 
institucionalización en los diferentes niveles educativos, etc. (Furco, 2011; Mayor, 2016; Puig, 2015).

El estudio científico de dicha temática ha permitido llegar a un consenso internacional que sirve para identificar las regularidades que estructuran esta práctica educativa (Montes, Tapia y Yaber, 2011): a) La planificación intencional e integrada de los objetivos curriculares o formativos y las acciones que conforman el servicio a la comunidad; b) El servicio tiene que estar dirigido a mejorar algún aspecto de la comunidad; y c) La participación fuerte de las personas en las distintas fases del proyecto: diseño, implementación y evaluación. Dichas señas de identidad son utilizadas para señalar las similitudes y límites que mantiene con otras prácticas educativas experienciales: voluntariado, trabajo de campo y acciones esporádicas comunitarias.

Las investigaciones realizadas también ponen en evidencia el potencial pedagógico de esta filosofía práctica, práctica educativa o metodología (Puig y Palos, 2006) para: influir positivamente en seis dimensiones relacionadas con el alumnado: desarrollo académico y cognitivo; desarrollo cívico; desarrollo vocacional y profesional; desarrollo ético y moral y desarrollo personal y social (Furco, 2007; Rodríguez y Ordoñez, 2015; Yorio y Feife, 2012)); impulsar la participación negociada del estudiantado-profesorado como estrategia para la mejora escolar (Fielding, 2011; Mayor y Rodríguez, 2017) y fortalecer las relaciones colaborativas entre los centros escolares y otras instituciones del entorno (Mayor, 2018).

El reconocimiento de las regularidades que sostienen al ApS como práctica educativa permiten, por un lado, ir dotándola de identidad como objeto de estudio científico y realizar investigaciones sobre los impactos que produce y, por otro, diferenciarla de otras prácticas educativas experienciales (voluntariado, trabajo de campo y acciones esporádicas comunitarias) que se vienen desarrollando en el ámbito comunitario.

En las actividades de ApS los aprendizajes, al estar vinculados con el servicio a la comunidad, favorecen la comprensión problemática de la realidad donde se va a actuar: diagnosticar y analizar las necesidades sociales a las que se pretende dar respuesta; implementar un servicio adecuado a dichas necesidades y reflexionar sobre lo acontecido. Todo ello favorece la significatividad, relevancia y utilidad de los aprendizajes, ya que son puestos en acción para clarificar y afrontar problemas básicos de la comunidad y para ampliar horizontes de conocimientos, sensibilidades y afectos. Desde esta perspectiva, lo que se aprende en las acciones de ApS se caracteriza por ser contextual, ya que parte de situaciones reales; por ser vivencial, porque requiere la implicación directa de los 
participantes en las distintas fases del proyecto; e integral, puesto que promueve el desarrollo de competencias necesarias para pensar y actuar sobre las realidades donde se ha decidido incidir (Díaz, 2015).

Las competencias, siguiendo a Pérez (2012), implican un saber pensar, decir, hacer y un querer. Constituyen el entramado básico, integrado por conocimientos, habilidades, actitudes y valores que utilizan las personas para comprender e intervenir en los distintos ámbitos de la vida personal, social y profesional. A diferencia de los aprendizajes memorísticos, descontextualizados y de escasa relevancia personal y social, requieren, para su puesta en acción, abordar tareas complejas y desafíos que inciten a los participantes a movilizar sus recursos y a construir otros para intentar dar respuestas a las nuevas situaciones problemáticas que afronta.

En el universo educativo, el ApS, se puede implementar en el ámbito de la educación formal (desde infantil a la universidad), así como en el ámbito de la educación no formal, dado que puede mejorar los procesos de aprendizaje al conectarlos a situaciones de la vida real que invitan a la reflexión-acción y permite el desarrollo de competencias para la vida atendiendo a las diferentes dimensiones de los individuos y grupos humanos.

Las competencias para la vida, siguiendo los planteamientos de la Comisión Europea (2010, p. 5), están orientadas al desarrollo de tres dimensiones:

a. realización y desarrollo personal a lo largo de la vida (capital cultural): las competencias clave deben permitir a las personas perseguir objetivos personales en la vida, llevados por sus intereses personales, sus aspiraciones y el deseo de continuar aprendiendo a lo largo de la vida; b. inclusión y una ciudadanía activa (capital social): las competencias clave deberían permitir a todos una participación como ciudadanos activos en la sociedad, y c. aptitud para el empleo (capital humano): la capacidad de todas y cada una de las personas de obtener un puesto de trabajo decente en el mercado laboral.

El estudio de múltiples prácticas de ApS (Deeley, 2016; Mayor, 2016; Montes, Tapia y Yaber, 2011; Rodríguez y Ordoñez, 2015; Rubio y Escofet, 2017; Yorio y Feife, 2012) ha puesto en evidencia que el alumnado que participa en las mismas puede adquirir e incrementar las siguientes competencias.

La Tabla 1 refleja un escenario amplio de posibles competencias y aprendizajes que los protagonistas de las prácticas de ApS pueden adquirir. Los mismos habrá que 
planificarlos y adaptarlos en función de las capacidades iniciales de los individuos y grupos, los objetivos del proyecto, el contexto donde se implementa, los avatares del proceso, etc., sin perder de vista, que una de las potencialidades pedagógicas del trabajo educativo por competencias reside en la necesidad de considerar que todos los elementos que la conforman (conocimientos, habilidades, actitudes y valores) "actúan dentro de un sistema que les da sentido, y sin el cual no puede entenderse la complejidad ni de la comprensión, ni de la actuación humana" (Pérez, 2012, p. 147).

\section{Tabla 1}

\section{Competencias y aprendizajes desarrollados en las prácticas de Aprendizaje-Servicio}

Competencias personales: están orientadas a la adquisición de aprendizajes que favorezcan el desarrollo de las capacidades cognitivas, afectivas, comportamentales y relacionales, para actuar de forma autónoma y responsable.

- Autoconocimiento y autoestima.

- Autonomía.

- Compromiso y responsabilidad.

Competencias para el pensamiento reflexivo, crítico y creativo: favorecen los aprendizajes pertinentes para comprender y valorar la complejidad del medio social y su implicación en el mismo.

- Curiosidad y motivación ante una realidad compleja y cambiante.

- Conciencia y comprensión de retos y problemas sociales complejos.

- Análisis y síntesis de la información, revisión de causas y consecuencias.

- Conexión del aprendizaje con la experiencia personal.

Competencias para la realización de proyectos: posibilitan la adquisición de herramientas congruentes para diseñar, implementar y evaluar acciones enfocadas a mejorar la realidad.

- Imaginación y creatividad para el diseño de proyectos.

- Implicación para planificar, organizar, desarrollar y evaluar propuestas.

- Reflexividad en los procesos, conclusiones y posibilidades de mejora.

- Difusión y transferencia de ideas y proyectos.

Competencias sociales y para la transformación social: impulsan el logro de aprendizajes para participar activamente, en defensa de los bienes comunes, como ciudadanos responsables.

- Participación responsable en la comunidad y asuntos públicos.

- Compromiso con el servicio comunitario.

- Importancia de la igualdad, justicia social y necesidad de cambio.

Competencias vocacionales y profesionales: aportan aprendizajes que capacitan para el desempeño en el mundo laboral.

- Conciencia de las opciones vocacionales.

- Preparación para el mundo del trabajo.

Fuente: elaboración propia a partir del trabajo de Rubio (2009).

\section{Metodología}

Siguiendo a Taylor y Bogdan (1992), en el ámbito de las ciencias sociales podemos encontrar dos enfoques (positivista y fenomenología) que representan dos formas distintas de mirar y significar la realidad desde una perspectiva ontológica, epistemológica y metodológica y supone un posicionamiento teórico y ético diferente por parte del investigador. 
Desde la perspectiva fenomenológica, también denominada como cualitativa, humanista, interpretativa, etc. (Wood y Smith, 2018), se concibe el mundo como algo inacabado, en constante construcción en tanto los sujetos que lo viven son capaces de modificarlo y darle significado. Para este enfoque, los sujetos y su manera de ver el mundo, el significado que éstos atribuyen a los fenómenos de estudio, es lo que constituye la realidad y lo que es importante estudiar (Taylor y Bogdan, 1992) en el contexto donde acontecen. Los diseños de investigación son abiertos, flexibles y emergentes para poder reformularse en función de la evolución de la investigación.

De los diferentes métodos cualitativos, de carácter exploratorio, hemos considerado la elección del estudio de caso intrínseco ${ }^{4}$ (Stake, 1999) como el adecuado debido a las siguientes razones: posibilita nuestra comprensión de la experiencia particular y compleja que, en este caso, vive un grupo de estudiantes que participaron en un proyecto de ApS en un contexto singular; favorece la reflexión de los diversos participantes en el estudio, así como la transferencia de los resultados a otros contextos, dependiendo del grado de similitud entre ambos (Guba, 1989, Pérez, Galán y Quintanal, 2012), y puede significar una oportunidad para modificar antiguas generalizaciones (Stake, 1999).

\subsection{Contexto de investigación}

El proyecto objeto de estudio supone una novedad, ya que es el primero que se realiza en la Facultad de Formación del Profesorado (Cáceres-España). Se llevó a cabo en el marco de la asignatura Organización y Gestión de Servicios Educativo-sociales, una de las materias obligatorias del tercer curso del grado de Educación Social.

Con dicha asignatura se prende pretende una aproximación a las instituciones educativo-sociales y especialmente a su dimensión organizativa y de gestión. Los aspectos que caracterizan este tipo de instituciones, su gestión y liderazgo y los componentes organizativos básicos (voluntariado, marketing, financiación...) se trabajaran de forma teórica, se analizaran y contrastaran en organizaciones concretas, a partir de trabajos de campo y casos prácticos, facilitando que la asignatura tenga un carácter aplicado (Universidad de Extremadura, 2017).

Este último asunto, su carácter aplicado, conecta con un tema que es objeto de

\footnotetext{
4 El estudio de casos intrínseco nos interesa porque con su estudio comprendemos en profundidad la particularidad de un fenómeno social. También puede servir de base para la elaboración de hipótesis (Stake, 1999).
} 
críticas recurrentes por parte del estudiantado que cursa este grado. Cuando se escucha su voz se puede apreciar el malestar generalizado, relacionado con la escasez de prácticas recogidas en el título del grado. Según los discentes, "el periodo de prácticas es insuficiente para nuestra correcta inserción laboral” (Ent ${ }^{5}$. A-10).

En línea con lo anterior, la posibilidad de implementar un proyecto de ApS conectaba fuertemente con el interés del alumnado, ya que "ofrecía una oportunidad para conocer de primera mano una organización socioeducativa y realizar un proyecto educativo con jóvenes" (Ent. A-5). Este es un asunto, que como se expondrá más adelante, incide significativamente en la motivación e implicación inicial del estudiantado en el proyecto.

\subsection{Participantes}

En la realización de dicha acción educativa participaron voluntariamente 24 de los 55 alumnos matriculados en la asignatura -los mismos conforman el conjunto de los informantes clave de la investigación-, 25 jóvenes de la Residencia "Universidad laboral" y 14 personas de la Asociación Síndrome de Down. Su implementación se materializó en una residencia, que acoge a estudiantes de secundaria, durante 7 sesiones de dos horas, en horario de tarde. Como tareas académicas encomendadas tuvieron que diseñar el proyecto, con la supervisión del profesor, y realizar un diario reflexivo con la intencionalidad de ir narrando los aspectos significativos que iban emergiendo, así como las potencialidades, debilidades y propuestas de mejora de los distintos aspectos que configuraban el proyecto. También asumieron la elaboración de un informe con los resultados de la investigación, que sería expuesto públicamente en clase y, posteriormente, en una mesa redonda que se desarrolló en la Facultad. Dichos instrumentos, diario reflexivo e informe de la investigación, se contemplaron como parte de la evaluación continua.

\subsection{Proceso de indagación e instrumentos utilizados}

El proceso de investigación se estructuró en cuatro fases (preparatoria, trabajo de campo, analítica e informativa) que se interrelacionaban unas con otras, pero siempre en un camino hacia adelante en el intento de responder a las preguntas de la investigación.

\footnotetext{
5 Abreviaturas utilizadas para referirnos a los distintos informantes y técnicas de recogida de datos: Est. (Estudiante); D. R. (Diario Reflexivo); Ent. (Entrevista); D.O. (Diario de Observación).
} 
Durante la realización de este se utilizaron las siguientes técnicas de recogida de datos:

- La observación participante se desarrolló en distintos espacios físicos de la Facultad (aulas y salón de actos) y en las diversas actividades que, derivadas del proyecto, se realizaron en la residencia del IES “Universidad Laboral”. Estás se fueron recogiendo en notas de campo que han constituido el diario de observación.

- El Análisis de documentos se centró en el proyecto de ApS, en los diarios reflexivos, en la guía de la asignatura, en los informes de investigación elaborados por los discentes y en el cuestionario ad hoc de tipo exploratorio diseñado por el profesor y contestado por 24 alumnos. El mismo contenía 32 preguntas relacionadas con cuatro dimensiones: competencias (conocimientos, habilidades, actitudes y valores que se pueden transferir a diversos contextos); servicio (conjunto de actividades conectadas a los aprendizajes curriculares orientadas a mejorar aspectos de la realidad); desarrollo personal-estudiantil (autoestima, responsabilidad, interés, dominio de contenidos, actitudes hacia el aprendizaje, ideas realistas sobre el mundo del trabajo, comunicación, trabajo en equipo, etc.); y desarrollo social (conocimiento de la realidad, convivencia, valores, interacción, análisis crítico de la realidad, compromiso social, etc.)

- La entrevista semiestructurada grupal. Fue diseñada con el propósito de contrastar algunas informaciones obtenidas durante el proceso de observación, análisis de documentos y resultados del cuestionario, así como ampliar algunos asuntos que emergían durante la indagación. El guion abierto, que orientaban a la misma, fue validado según el procedimiento de juicios de expertos para enfocar las cuestiones relevantes para la investigación (Wood y Smith, 2018). Fue dirigida a 3 grupos de 8 estudiantes.

Tanto las observaciones como las entrevistas realizadas fueron registradas en vídeo y, posteriormente, se realizó la transcripción literal de todo lo expresado y acontecido, presentándose la misma a los informantes para obtener su validación, responder a los principios básicos de la investigación ética: consentimiento, honestidad y el cuidado de las personas informantes, eliminar el sesgo del investigador e intentar asegurar la credibilidad y replicabilidad de los datos (Guba, 1989).

\section{Resultados y su análisis}


El sistema de categorización de los datos extraídos de las entrevistas, documentos y observaciones se ha configurado a partir de la identificación de núcleos de ideas con sentido completo relacionadas con las preguntas que orientaban la investigación. Nuestro enfoque para el análisis de datos cualitativos ha sido interpretativo, para ello realizamos la tarea básica de reducción de la información a través de categorizaciones ${ }^{6}$. El sistema de categorías ha sido inductivo pues al examinar los datos de las extraídos de los instrumentos nos preguntamos por el tópico más adecuado capaz de cubrir cada unidad, generando así las categorías y subcategorías.

En la Tabla 2 presentamos los resultados obtenidos agrupados por categorías (competencias personales-estudiantiles y competencias sociales) y subcategorías.

Tabla 2

Categorías y subcategorías fijadas en el análisis

\begin{tabular}{lll}
\hline Categorías & Subcategorías & Subcategorías \\
\hline Competencias & -Interés & -Autodescubrimiento. \\
personales- & -Afrontar miedos. & -Desarrollo competencias. \\
estudiantiles & -Compromiso personal. & -Aprendizajes significativos. \\
& -Desarrollo autonomía. & -Reflexión. \\
\hline Competencias & -Mayor conocimiento realidad. & -Toma de conciencia. \\
sociales & -Mayor compromiso cívico. & -Satisfacción trabajo.
\end{tabular}

Fuente: elaboración propia, 2018.

En los estudios de caso existen múltiples formatos para presentar los resultados obtenidos (Simons, 2011). En este caso hemos utilizado un texto narrativo con descripciones de los temas más significativos o subcategorías (Tabla 2) que sirvieron para la conformación de dos categorías. También se han incorporado algunos de los resultados alcanzados en el cuestionario ad hoc, ya que sirven para triangular algunas informaciones obtenidas en las entrevistas y diarios reflexivos. Cada una de las categorías, aunque se presentan separadas para favorecer la descripción detallada de los mismas, forman parte de la textura global de la experiencia analizada.

\subsection{Cuando el alumnado se coloca en posición de agente reflexivo y crítico puede tomar conciencia de su reconstrucción personal y estudiantil}

\footnotetext{
${ }^{6}$ La categorización temática implica la categorización de temas, en función de las preguntas del estudio, derivados de los datos obtenidos a través de los distintos instrumentos de recogida de información utilizados (Cisterna, 2005; Wood y Smith, 2018).
} 
El interés o motivación intrínseca del estudiantado es uno de los factores implicados en los procesos de enseñanza-aprendizaje en el ámbito académico. Las actitudes, percepciones, expectativas y representaciones que tenga el estudiantado de sí mismo, de las tareas a realizar, y de las metas que pretende alcanzar constituyen dimensiones significativas que orientan la conducta de este. En este sentido, el alumnado que participó en el proyecto estaba muy interesado en implicarse en acciones formativas que le permitiesen enredarse en contextos reales para realizar prácticas:

Llevamos tres años estudiando en el grado de Educación Social y todavía no hemos realizados ninguna práctica (Ent. Est.-6).

Tengo muchas ganas de conocer por dentro como funciona alguna asociación o institución donde se supone que luego vamos a trabajar (Ent. Est.-15).

Cuando el profesor nos propuso la posibilidad de realizar un proyecto de ApS a mí me encantó, ya que es lo que llevamos tiempo reclamando, que tenemos mucha teoría y poca práctica (Ent. Est.-7).

Dichas motivaciones están en línea con las valoraciones asignadas por los discentes en el cuestionario ad hoc. En este instrumento el ítem vinculado al nivel de interés que despertó su enganche en el proyecto (Figura 1) fue valorado como alto por el más del 29\% (7 de 24) de los participantes y como muy alto por más del 70\% (14 de 24). 
Figura 1. Grado de interés alcanzado por el alumnado que participó en el proyecto de ApS.

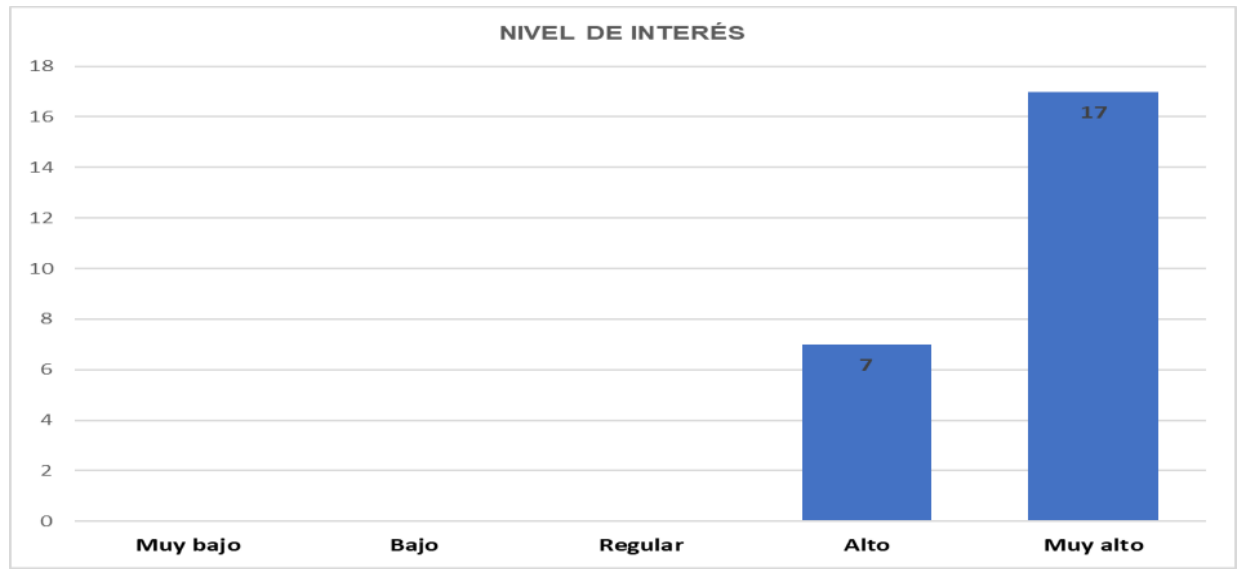

Fuente: elaboración propia, 2018.

Implicarse en acciones nuevas y contextos desconocidos en posición de agentes activos supuso una reconfiguración del rol que hasta ese momento venían desempeñando. Pasaron de "escuchar en clase a los profesores a pensarnos como futuros profesionales que teníamos que diseñar un proyecto para realizarlo con jóvenes" (Ent. Est.-10). Un proceso de aprendizaje que les posibilitó descubrir potencialidades y dificultades personales.

Algunas estudiantes relatan en sus diarios reflexivos que su implicación en el proyecto le había servido, entre otras cuestiones, para superar algunos miedos iniciales:

Fui muy insegura, sin saber con lo que me iba a encontrar, temiendo mis reacciones $y$ sus reacciones, ya que me considero una persona con mucho carácter. Sin embargo, creo que casi siempre, he estado a la altura, me he sabido comportar, he sabido dirigir, intervenir, e incluso ponerme firme cuando era necesario, aunque, todo se pueda realizar mucho mejor (D.R. Est-1).

El primer día que empecé me sentía con miedo y muy frustrada puesto que pensaba que no valía para llevar a cabo este tipo de tareas y, sin embargo, poco a poco fui acomodándome a la situación y adaptándome a lo que sucedía, hasta que me he dado cuenta de que puedo valer para todo aquello que me proponga como persona y como profesional, puesto que mis sentimientos durante las varias semanas que duró el proyecto fueron cambiando de negativos a positivos progresivamente (D.R. Est-5). 
Como plantea Freire (1983), la capacidad de aprendizaje del ser humano, su desarrollo indeterminado, la naturaleza compleja de las relaciones, etc., son algunas de las cuestiones que hacen posible que nuestro ser, estar y sentir se vaya modificando, ya que nada está dado de una vez y para siempre.

Situar al estudiantado en contextos reales de enseñanza que le son ajenos ya es de por sí un elemento innovador que invita a poner en acción conocimientos y saberes procedentes de su vida académica y/o experiencia personal, a entender la duda como elemento pedagógico que ayuda a la adquisición o reconstrucción de nuevas formas de comprender o afrontar la realidad. Un proceso que invita al despliegue de la autonomía, al descubrimiento personal y a poner en valor algunos aprendizajes alcanzados en el ámbito universitario:

Me ha ayudado a abrirme con las personas y a ser menos seria. Pienso que he tenido un cambio de manera progresiva, ya que no fue del tirón, sino que poco a poco fui dejando atrás la vergüenza, el tener miedo al hablar en público y abrirme más con las personas (Ent. Est.-23).

Participar en el proyecto nos ha enseñado a afrontar los problemas y que, aunque exista un currículum planteado y organizado, tenemos que aprender a tener alternativas para poder conseguir nuestro objetivo con éxito. Este caso nos pasó a nosotros (grupo B) al haber calculado mal el tiempo de las actividades, teniendo que incorporar otra actividad más para así poder rellenar el gran hueco que nos quedaba (...) (Ent. Est.-8).

Cuando el estudiantado se engancha en acciones reflexivas, a las que pueden dotar de sentido y significado, su nivel de satisfacción es muy elevado:

Creo que es una forma increíble de aprender y de educar, ya que se educa mediante la práctica y dándole total responsabilidad al alumnado, haciendo que se involucren mucho más y que se tomen las actividades más en serio. Para mí, ha sido una forma nueva de aprendizaje y me ha gustado muchísimo, me encantaría que se llevará más a cabo pues es una experiencia que te marca en muchos aspectos de tu vida, no solo en el educativo (Ent. Est.-16). 
Me siento muy afortunada de haber realizado este proyecto y de todo lo que he aprendido en él, lo repetiría muchas veces más y me alegro de haber tomado esta decisión (D.R. Est.-4).

Es una de las mejores experiencias vividas en el grado de Educación Social, me ha aportado múltiples conocimientos y creo que se ha realizado un proyecto de ApS donde se ha generado un impacto social y, al mismo tiempo, he adquirido competencias muy útiles (D.R. Est.-3).

Durante el proceso nos dimos cuenta de que el proyecto que realizábamos no nos venía impuesto desde fuera, sino que éramos los auténticos protagonistas del mismo. Ello me llenó de satisfacción (Ent. Est.-10).

Las afirmaciones y valoraciones positivas anteriores, planteadas por el estudiantado en las entrevistas y diarios reflexivos, son congruentes con el nivel de satisfacción alcanzado en el cuestionario ad hoc (Figura 2). El ítem relacionado con este aspecto ha sido valorado como alto por más del $16 \%$ (4 de 24 ) y como muy alto por más del $83 \%$ (20 de 24 ) del alumnado implicado.

Figura 2. Nivel de satisfacción alcanzado por el alumnado que participó en el proyecto de ApS.

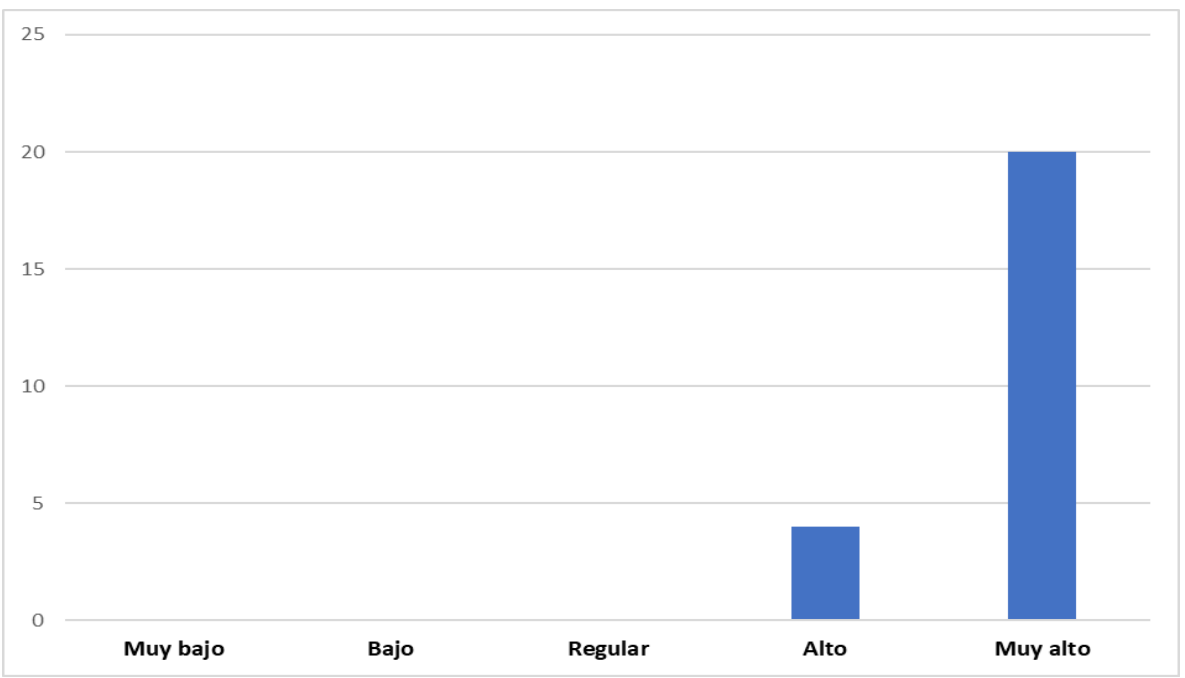

Fuente: elaboración propia, 2018. 


\subsection{El servicio a la comunidad posibilita que el alumnado desarrolle competencias sociales y dote de sentido los contenidos curriculares}

Según el Ministerio de Educación, Cultura y Deporte (2006), la competencia social se refiere a la capacidad de comprender la realidad social en que se vive, de cooperar, convivir y ejercer la ciudadanía democrática en una sociedad plural, así como comprometerse en su mejora. Una concepción que conecta con los ejes que estructuran las prácticas de ApS, ya que los aprendizajes, al estar imbricados con el servicio, favorecen la comprensión problemática de la realidad donde se va a actuar: diagnosticar y analizar las necesidades sociales a las que se pretende dar respuesta, implementar un servicio adecuado a dichas necesidades y reflexionar sobre lo acontecido. Todo ello aumenta el conocimiento de la realidad

Participar en el proyecto me ha ofrecido una oportunidad excelente para conocer un ámbito donde trabajan educadores sociales, una oportunidad de conectar la teoría que veíamos en clase con la práctica, de obtener experiencias que me pueden servir para mi futuro profesional, etc. (Ent. Est-16).

Los proyectos de ApS están orientados a promover el aprendizaje experiencial que permite a los estudiantes vincular el pensamiento a la acción, teoría y práctica, y pretenden desarrollar en ellos la capacidad de construir, aplicar y transferir el conocimiento al enfrentarlos a la vida real con la intencionalidad de mejorar aspectos de esta. En esta línea, los estudiantes han corroborado en las entrevistas que esta metodología permite vincular los contenidos teóricos a la práctica, promueve una mayor formación práctica y la reelaboración de los contenidos teóricos para hacerlos más pertinentes al contexto social para el desempeño profesional.

Se nos han presentado situaciones que no sabíamos cómo resolver y hemos tenido que hacer eso que tantas veces nos planteaban los profesores: repensar y reestructurar las cosas de la manera más rápida y eficaz posible. Un día nos sobraba tiempo, otro día nos faltaba, otro día nos encontrábamos con chicos que se negaban a participar o que no estaban nada motivados con las actividades (...) (Ent. Est.-9).

He aprendido la importancia de reflexionar sobre las actividades que realizamos, ya que nos ayuda a adquirir nuevos conocimientos, nos abre dudas, y nos ayuda a mejorar nuestras acciones (Ent. Est.-15). 
Para diseñar y poder realizar el proyecto, hemos tenido que incorporar parte de los contenidos teóricos tratados en la asignatura, pero me ha gustado ver su aplicación práctica a la realidad (Ent. Est.-21).

Durante la presentación pública de los resultados del informe elaborado, los representantes del alumnado que había participado en el proyecto de la Residencia "Universidad Laboral" manifestaron que 15 de ellos seguirían como personas voluntarias en dicha organización (D.O.). Un compromiso cívico que muestra las virtualidades pedagógicas de las prácticas de ApS para, entre otras cuestiones, generar espacios de encuentro entre el estudiantado y los escenarios (entidades sociales, instituciones públicas y empresas privadas) donde posiblemente desarrollarán su profesión, conocer el trabajo de los profesionales que trabajan en dichos escenarios y reconstruir sus saberes y formas de participar en asuntos socioeducativos:

Con la participación en este proyecto hemos descubierto un mundo que no sabíamos que existía, eso ha despertado mi interés por profundizar en el tema de la residencia de jóvenes y por participar en organizaciones sociales. (...). No sabía cómo se organizaban, ni que tuvieran tantos problemas económicos para llevar a cabo sus actividades (Ent. Est.-22).

Para evaluar el nivel alcanzado en la adquisición de algunas competencias específicas de la asignatura, el profesor utilizó distintos instrumentos: informe reflexivo, exposición pública de los resultados obtenidos, participación en la mesa redonda, trabajo creativo sobre las organizaciones sociales y el cuestionario ad hoc (D.O.). En el cuestionario ad hoc planteó varias preguntas vinculadas al nivel alcanzado en algunas de las competencias específicas de la asignatura. Una de ellas recogía lo siguiente: En relación con la competencia específica Diseñar planes, programas y proyectos de actividades de intervención socioeducativa en diversos contextos, ¿qué nivel de competencia consideras que has alcanzado? (Figura 3). En este sentido, más del 37\% (9 de 24) del estudiantado implicado en el proyecto respondía que alto, mientras más del 62\% (15 de 24) valoraba que muy alto. 
Figura 3. Nivel alcanzado por el estudiantado en la competencia 3.

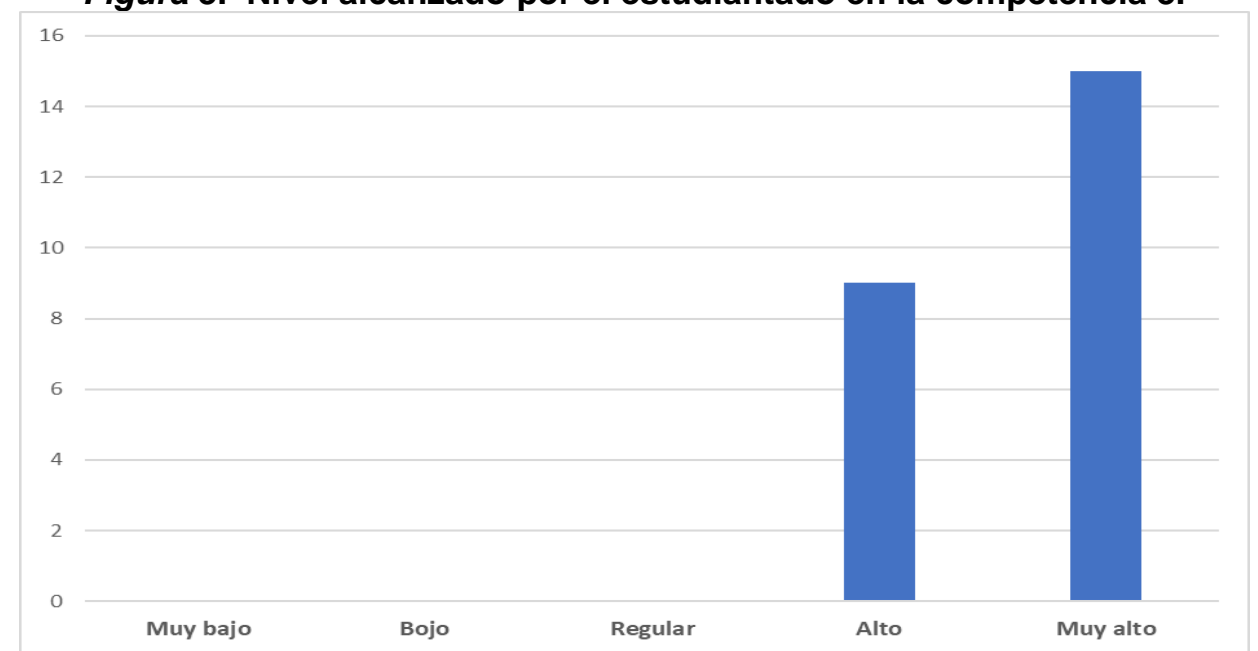

Fuente: elaboración propia, 2018.

\section{Conclusiones}

El presente estudio nació con el propósito de indagar cómo incide un proyecto de ApS en el desarrollo de competencias personales-estudiantiles y sociales del estudiantado universitario.

Desde una aproximación cualitativa de análisis de contenido sobre la categoría competencias personal-estudiantiles, han emergido las subcategorías expuestas en la Tabla 2. Las mismas se hallaban, en muchos casos, imbricadas, retroalimentándose mutuamente. Así se pudo observar que el interés inicial, basado fundamentalmente en el deseo de conocer contextos reales y poder intervenir en los mismos, despertaba miedos en algunos discentes, pero una vez afrontados servían de base para el desarrollo de su autoestima y una mayor implicación en el proyecto. En este sentido, y a partir de los hallazgos alcanzados, se puede concluir que las prácticas de ApS influyen de forma significativa en el desarrollo de competencias personales-estudiantiles, siendo notable la mediación de las subcategorías: interés inicial, compromiso fuerte del alumnado en las distintas fases del proyecto, los momentos de reflexión y la satisfacción obtenido por el trabajo realizado. Algunos de estos resultados son coincidentes con los planteados por Furco (2003). En su estudio plantea que la motivación intrínseca o interés, junto con la autoestima, el empoderamiento, la conducta prosocial y el compromiso actúan como factores mediadores del éxito académico.

También están en línea con las conclusiones alcanzadas por Páez y Puig (2013), los cuales señalan la importancia de la reflexión como elemento mediador en las prácticas de ApS, con la intencionalidad educativa de favorecer la implicación del estudiantado en la 
problematización de su experiencia.

Otro asunto significativo es el alto nivel de satisfacción alcanzado por el alumnado. La noción satisfacción o sentimiento de bienestar puede ser considerada como un constructo borroso de naturaleza multidimensional que incluye componentes objetivos (características de los proyectos de ApS y contextos educativos donde se implementan) y subjetivos relacionados con la atribución de significados (conocimiento y utilidad atribuida, valoración del proceso y resultados, etc.). Desde esta perspectiva, los resultados obtenidos la experiencia investigada nos llevan a concluir que en el alto nivel de satisfacción alcanzada inciden varias dimensiones: la posibilidad de transferir los contenidos curriculares a situaciones de la vida real, el compromiso fuerte del estudiantado en las distintas fases del proyecto, la creación de contextos formativos donde se produzcan situaciones problemáticas que ayuden al alumnado a afrontar algunas dificultades personales, la utilidad atribuida al conocimiento adquirido y los momentos de reflexión después de la acción. Algunos de dichos resultados son coincidentes con los hallados en la investigación implementada por González y Marta (2015): satisfacción por la transferencia de los contenidos curriculares a situaciones de la vida real.

En cuanto a la categoría competencias sociales, el ApS invita a observar, analizar e intervenir sobre la realidad, ello implica un mayor conocimiento de distintos ámbitos de la realidad, favorece la toma de conciencia a través del análisis de los factores que están incidiendo en la misma e invita a la puesta en acción de actividades que promueven el compromiso cívico, entendido éste como las acciones individuales y colectivas encaminadas a identificar y abordar cuestiones de interés público. Dichas apreciaciones son coincidentes con los resultados hallados en las investigaciones realizadas por Furco (2003), Rodríguez y Ordoñez (2015) y Yorio y Feife (2012). En las mismas se plantean que el ApS promueve el desarrollo de diversas dimensiones relacionadas con el compromiso cívico: mayor participación en la comunidad, mayor ejercicio de ciudadanía y mayor conciencia de cuestiones sociales.

Teniendo en cuenta lo expuesto anteriormente, podemos concluir planteando que las prácticas de ApS se configuran como escenarios formativos con notables virtualidades pedagógicas para favorecer la adquisición de competencias personales-estudiantiles y sociales necesarias para diagnosticar e intervenir en la realidad, diseñar actividades reflexivas encaminadas a mejorar aspectos de esta y potenciar el compromiso personal y social del estudiantado universitario en la mejora de las necesidades sociales identificadas. 
En relación con los límites de este estudio y su posible aportación de la comunidad científica, cabe señalar que una limitación es su carácter de estudio de caso a partir de un fenómeno singular, por lo que no se pretende la generalización de sus resultados a otros casos, aunque los resultados pueden significar una nueva oportunidad para modificar antiguas generalizaciones (Stake, 1999).

En cuanto a posibles líneas de investigación, se propone avanzar en las siguientes cuestiones:

- Indagar las razones que sustentan la participación de los distintos agentes implicados en las prácticas de ApS.

- Estudiar el tipo y niveles de participación que promueven los proyectos de ApS.

- Explorar la influencia de esta experiencia en la formación del alumnado universitario a medio-largo plazo.

- Analizar la influencia de los proyectos de ApS en los cambios y mejoras producidas en el centro educativo.

\section{Referencias}

Cisterna, Francisco. (2005). Categorización y triangulación como procesos de validación del conocimiento en investigación cualitativa. Theoria, 4(1), 61-75. Recuperado de http://www.redalyc.org/pdf/299/29900107.pdf

Coll, Cesar. (2007). Las competencias en la educación escolar: algo más que una moda y mucho menos que un remedio. Aula de Innovación Educativa, 161, 34-39.

Comisión Europea. (2010). Competencias clave para un aprendizaje a lo largo de la vida. Dirección General de Educación y Cultura. Recuperado de http://www.educastur.princast.es/info/calidad/indicadores/doc/comision europea.pdf

De Miguel, Mario. (2006). Metodologías para optimizar el aprendizaje. Segundo objetivo del Espacio Europeo de Educación Superior. Revista Interuniversitaria de Formación del Profesorado, 20(3), 71-91. Recuperado de http://www.redalyc.org/pdf/274/27411311004.pdf

Deeley, Susan. (2016). Aprendizaje-Servicio en educación superior. Teoría, práctica y perspectiva crítica. Madrid, España: Narcea.

Dewey, John. (1950). Las escuelas del mañana. Buenos Aires, Argentina: Losada.

Díaz, Frida. (2015) Estrategias para el desarrollo de competencias en Educación Superior, En Gabriela, Carrillo Mendoza (Ed.), I Encuentro Internacional Universitario. El currículo por competencias en la Educación Superior (pp.63-86). Pontificia Universidad Católica del Perú, Lima, Perú. Recuperado de http://cdn02.pucp.education/academico/2015/09/01094909/curriculo competencias.pdf 
Fielding, Michael. (2011). La voz del alumnado y la inclusión educativa: una aproximación democrática radical para el aprendizaje intergeneracional. Revista Interuniversitaria de Formación del Profesorado, 25(1), 31-61. Recuperado de http://www.redalyc.org/pdf/274/27419147003.pdf

Freire, Paulo. (1983). La educación como práctica de la libertad. Madrid, España: Siglo XXI.

Furco, Andrew. (2003). El impacto educacional del Aprendizaje-Servicio. ¿Qué sabemos a partir de la investigación? [pdf]. Recuperado de https://roserbatlle.files.wordpress.com/2009/02/resultats-investigacio-aps-furco-modode-compatibilidad.pdf

Furco, Andrew. (2007). Impacto de los proyectos de aprendizaje-servicio. En A. González (coord.), Seminarios Internacionales de Aprendizaje y Servicio Solidario (pp.175-183). Buenos Aires, Argentina: EUBEDA.

Furco, Andrew. (2011). El aprendizaje-servicio: un enfoque equilibrado de la educación experiencial. Revista Educación Global, 0, 64-70. Recuperado de http://educacionglobalresearch.net/furco1issuezero

González, Patricia y Marta, Carmen. (2015). La metodología del aprendizaje-servicio como herramienta en la formación de los periodistas. Opción, 31(3), 564-581. Recuperado de http://www.redalyc.org/pdf/310/31045567029.pdf

Guba, Egon. (1989). Criterios de credibilidad en la investigación naturalista. En J. Gimeno y A. I. Pérez. (coords.). La enseñanza: su teoría y su práctica (pp. 148-165). Madrid, España: Akal.

Imbernón, Francesc y Medina, José Luis. (2008). Metodología participativa en el aula universitaria. La participación del alumnado. Barcelona, España: Octaedro.

James, William. (1910). The Works of William James. En Mónica, Aguerri (Ed. y Trad.), El equivalente moral de la guerra. Recuperado de http://www.unav.es/gep/TheMoralEquivalentOfWar.html

Mayor, Domingo. (2016). El Aprendizaje-Servicio como práctica educativa que promueve relaciones colaborativas entre la escuela y la comunidad (Tesis doctoral). Universidad de Almería, España.

Mayor, Domingo. (2018). Prácticas de Aprendizaje-Servicio como escenarios de confluencia entre la educación escolar y social. Revista Iberoamericana de Educación, 76, 35-56. Recuperado de https://rieoei.org/RIE/article/view/2847

Mayor, Domingo y Rodríguez, Dolores. (2017). Aprendizaje-Servicio: una práctica pedagógica que promueve la participación del estudiantado para la mejora escolar y social. Revista Complutense de Educación, 28(2), 555-571. Recuperado de http://revistas.ucm.es/index.php/RCED/article/view/49623 
Ministerio de Educación, Cultura y Deporte. (2006). Real Decreto 1513/2006 por el que se establecen las enseñanzas mínimas para la Educación primaria. Gobierno de España. Recuperado de https://www.boe.es/boe/dias/2006/12/08/pdfs/A43053-43102.pdf

Montes, Rosalía, Tapia, María Nieves y Yaber, Lorena. (2011). Manual para docentes y estudiantes solidarios. Buenos Aires, Argentina: CLAYSS. Recuperado de http://www.clayss.org.ar/04 publicaciones/Natura2013.pdf

Páez, Mireia y Puig, José María. (2013). La reflexión en el Aprendizaje-Servicio. Revista Internacional de Educación para la Justicia Social, 2(2), 13-32. Recuperado de https://repositorio.uam.es/bitstream/handle/10486/660355/art1.pdf?sequence $=1$

Pérez, Miguel Ángel. (2012). Educarse en la era digital. Madrid, España: Morata.

Pérez, Ramón, Galán, Arturo y Quintanal, José. (2012). Métodos y diseños de investigación en educación. España: UNED-Universidad Nacional de Educación a Distancia.

Puig, José María. (coord.). (2015). 11 ideas clave. ¿Cómo realizar un proyecto de aprendizaje-servicio?. Barcelona, España: Graó.

Puig, José María y Palos, Josep. (2006). Rasgos pedagógicos del aprendizaje-servicio. Cuadernos de Pedagogía, 357, 60-63.

Rodríguez, Margarita y Ordoñez, Rosario. (2015). Una experiencia de Aprendizaje-Servicio en comunidades de aprendizaje. Profesorado. Revista de Currículum y Formación del Profesorado, 19(1), 314-333. Recuperado de https://recyt.fecyt.es/index.php/profesorado/article/view/41039

Rubio, Laura. (2009). El aprendizaje en el aprendizaje servicio. En J. M. Puig (coord.). Aprendizaje servicio. Educación y compromiso cívico (pp. 91-105). Barcelona, España: Graó.

Rubio, Laura y Escofet, Anna (coords.). (2017). Aprendizaje-Servicio (ApS): claves para su desarrollo en la Universidad. Barcelona, España: Octaedro.

Simons, Helen. (2011). El estudio de caso: Teoría y práctica. Madrid; España: Morata.

Stake, Robert. (1999). Investigación con estudio de casos. Madrid, España: Morata.

Taylor, Stephen y Bogdan, Robert. (1992). Introducción a los métodos cualitativos de investigación. Barcelona, España: Paidós.

Universidad de Extremadura. (2017). Guía de la asignatura Organización y Gestión de Servicios Educativo-sociales. Facultad de Formación del Profesorado: Autor.

Wood, Phil y Smith, Joan. (2018). Investigar en educación. Conceptos básicos y metodología para desarrollar proyectos de investigación. Madrid, España: Narcea.

Yorio, Patrick y Feifei, Ye. (2012). A Meta-Analysis on the Effets of Servie-learning on the Social, Personal, and Cognitive Outcones of Learning. Academy of Management Learning \& Education, 11(2), 9-27. Recuperado de https://journals.aom.org/doi/abs/10.5465/amle.2010.0072?journalCode=amle 